\title{
„Kunst am Bau“ in der Württembergischen Landesbibliothek Stuttgart
}

\author{
Von Vera Trost
}

Die Württembergischen Landesbibliothek Stuttgart begeht am 11. Februar 2015 ihren 250. Geburtstag. Sie strebt zu diesem Jubiläum die Eröffnung eines seit langem geplanten Erweiterungsbaus an, um den Anforderungen einer modernen wissenschaftlichen Bibliothek und eines lebendigen Lern- und Kulturzentrums im 21. Jahrhundert gerecht zu werden. Ihre französische Partnerbibliothek, die Bibliothèque nationale et universitaire de Strasbourg (BNU), hat die Planungsphase ihrer Erweiterung und Modernisierung gerade hinter sich gelassen und befindet sich nun mitten im Umbau. Die erste Ausgabe von "La Revue de la BNU“ beschäftigt sich deshalb mit dem Thema „Bibliothèques et identité visuelle“, zu dem die WLB mit einem Beitrag über die „Kunst am Bau“ und die Grafiken Josua Reicherts eingeladen wurde.

Der vorliegende Beitrag ist mit dem Artikel „L'art dans l'architecture à la Bibliothéque régionale du Wurtemberg : l'oeuvre graphique de Josua Reichert" nicht ganz identisch, sondern enthält darüber hinaus Angaben zu Kunstwerken, die in den 1960er Jahren von anderen Künstlern für die WLB erworben wurden.

\section{Die Württembergische Landesbibliothek}

Die Württembergische Landesbibliothek (WLB) wurde am 11. Februar 1765 von Herzog Carl Eugen von Württemberg als öffentliche Bibliothek in der damaligen Residenzstadt Ludwigsburg gegründet. 1777 wurde sie nach Stuttgart verlegt und erhielt 1883 am heutigen Standort Konrad-Adenauer-Straße ein neues Gebäude - zu jener Zeit der zweitgrößte Bibliotheksbau Süddeutschlands. Dieses Gebäude brannte in der Nacht vom 12./13. September 1944 zusammen mit über 500.000 Büchern fast vollständig ab. Zwar wurde es sofort nach Kriegsende unter dem neu ernannten Direktor Dr. Wilhelm Hoffmann noch behelfsmäßig genutzt, letztlich entschloss man sich aber doch nicht zu einem Wiederaufbau, sondern zu einem Neubau am alten Standort Konrad-Adenauer-Straße.

\section{Der Bibliotheksbau}

Direktor Hoffmann legte ein Konzept vor, nach dem in einem offenen, den vielfältigen Aufgaben entsprechenden Gebäude alle Bürger des Landes Zugang zur Welt der Bücher haben sollten. Das in der Bibliothek gesammelte "Gedächtnis der Menschheit" sollte nicht nur in den verschiedenen Lesesälen und Magazinen bereitstehen, sondern auch in einem „Buchmuseum" vermittelt werden.

Die Architekten unter der Leitung von Professor Horst Linde nahmen dieses Konzept auf und begriffen die Bibliothek einerseits als eine Stätte der Informationsvermittlung und andererseits vor allem auch als einen sozialen Ort und gesellschaftlichen Treffpunkt. Dies sollte sich in der Architektur widerspiegeln und kraftvoll ausstrahlen. Wie aktuell diese Überlegungen noch heute, etwa ein halbes Jahrhundert später, sind, zeigt sich, wenn man Foyer, Lesesäle, "Buchmuseum" und die angeschlossene Cafeteria betritt und sie als überaus lebendigen Lernort und aktive Kultureinrichtung wahrnimmt.

Eine weitere Forderung war, die Bibliothek in den Stadtraum zu integrieren. Deshalb führte man die Fußgängerwege durch „bewohnbare“ Grünanlagen - ähnlich den benachbarten Theateranlagen - auf die Bibliothek zu. Das Kopfsteinpflaster der Wege wurde der Belag für die Rampe, die im Gebäude den Eingang mit dem Foyer verbindet. Somit schreitet der 
Besucher unmerklich aus dem Garten in die Bibliothek hinein, wo sich in der "gegliederten Landschaft des Innenraums" die Gartenanlage fortsetzt und wiederum über Stufungen, Terrassen und große Glasflächen den Blick über den großräumigen Stadtraum hin zum begrünten Rand des Talkessels und den Weinbergen in der Ferne freigibt.

Der Besucher gelangt über drei Eingänge auf zwei Ebenen in das großzügige transparente Treppenhaus und erreicht ohne Hindernis Buchmuseum, Cafeteria, Foyer, Information und Leihstelle. Nur die Lesesäle wie auch die Diensträume wurden durch Türen vom öffentlichen Bereich getrennt, um dort ungestörtes und konzentriertes Arbeiten zu ermöglichen.

\section{Kunst am Bau}

Das neue Gebäude wurde 1970 bezogen. Doch bereits in den 60er Jahren hat man für den Neubau der Landesbibliothek künstlerische Arbeiten erworben.

Die Grundlage dafür war die Bekanntmachung der Landesregierung über die Förderung der bildenden Kunst und des Kunsthandwerks vom 2. Mai 1955:

„Zur Förderung des bildenden Kunst und des Kunsthandwerks wird die Regierung des Landes Baden-Württemberg bei allen staatlichen Bauaufträgen einen Beitrag für bildnerische und kunsthandwerkliche Arbeiten vorsehen, die im Regelfall 1 bis $2 \%$ der Bauauftragsumme beträgt.

Die Landesvermögens- und Bauabteilungen bei den Oberfinanzdirektionen entscheiden im Benehmen mit dem Finanzministerium und den zuständigen Fachverwaltungen. Bei der Vergebung der Aufträge werden geeignete Sachverständige zugezogen und die Verbände bildender Künstler gehört." (Regierung des Landes Baden-Württemberg, veröffentlicht in Stuttgart, den 14. Juni 1955 in: Gemeinsames Amtsblatt des Landes Baden-Württemberg, hrsg. vom Innenministerium, Ausgabe A, 3. Jahrgang, Nr. 13)

\section{Kunstkommission}

Die sog. 2\%-Klausel zu Kunst und Kunsthandwerk im Bereich des Staatlichen Hochbaus ist noch heute gültig. Die Auswahl der Kunstwerke und Künstler wird immer noch von der „Kunstkommission“ vorgenommen, die bei Vermögen und Bau Baden-Württemberg angesiedelt ist. Sie setzt sich zusammen aus Vertretern der Staatlichen Hochbauverwaltung und der nutzenden Verwaltung, freischaffenden Künstlern und gegebenenfalls dem freien Architekten und hat die Aufgabe, Empfehlungen für künstlerische Aufgabenstellungen zu erarbeiten und die Auswahl des Verfahrens zu treffen. Es kommen dafür beschränkte öffentliche Wettbewerbe oder eine Direktbeauftragung in Frage. Die Auswahl der Künstler und die Nominierung der Jury sowie die Beurteilung der künstlerischen Arbeiten werden in der Kunstkommission getroffen.

\section{Kunstwerke}

Vor Fertigstellung des Neubaus wurden folgende Kunstwerke erworben:

Magdalena Abakanowicz, Wandteppich „Gewebe 24 in rot und scharz“ 1963 (300 x 413 $\mathrm{cm})$

Das Werk der polnischen Textilkünstlerin und Bildhauerin Abakanowicz (geb. am 20. 6. 1930 Falenty bei Warschau) wurde im November 1964 von der Galerie Pauli, Lausanne für 18.000 DM erworben. Bis zur Inbetriebnahme der Landesbibliothek befand sich der Wandteppich im Finanzministerium, heute hängt er zentral im Foyer am Übergang zur Direktion. 
Alexander Camaro, „Monsum“ (Öl auf Leinwand, $160 \times 180 \mathrm{~cm}$ )

Das Finanzministerium stimmte am 26.5.1965 zu, das Werk des Berliner Malers und documenta-Teilnehmers Camaro (geb. 27. September 1901 in Breslau, gest. 20. Oktober 1992 in Berlin) für 14.500 DM anzukaufen.

Das Gemälde hängt heute auf der Galerie an der Wand zur Direktion.

Hans Martin Erhardt, Pastellbilder „Stilleben 4“ und „Stilleben 13“

Die Pastelle des Karlsruher Malers und Graphikers (geb. 28. Oktober 1935 in Emmendingen, u.a. Schüler HAP Grieshabers) wurden im Juni 1965 für je 600 DM beschafft. Die Bilder wurden inzwischen an die Abteilung Vermögen und Bau Baden-Württemberg zurückgegeben.

\section{Rolf Nesch, „San Marco, Venedig“ (6 Metalldrucke)}

Die Drucke des deutsch-norwegischen Malers und Grafikers Nesch (geb. 7. Januar 1893 in Oberesslingen am Neckar, gest. 27. Oktober 1975 in Oslo) wurden nach Absprache zwischen dem Finanzministerium und der Landesbibliothek von der Galerie Franke, München zum Preis von 12.500 DM erworben. Übergangsweise wurde die Arbeit im Finanzministerium aufbewahrt, heute hängt sie im Zimmer des Direktors.

\section{Henry Moore, Shelter Sketch-Book, Berlin und London 1967}

Das Skizzenbuch - deutsche Ausgabe 9 - des englischen Bildhauers und Zeichners Moore (geb. 30. Juli 1898 in Castleford, Yorkshire, gest. 31. August 1986 in Much Hadham, Hertfordshire) wurde vom Rembrandt Verlag in Berlin zum Preis von 1.024,- DM erworben. Die 80 Zeichnungen in Faksimiledrucken und eine Originallithographie waren auf besonderen Wunsch der Landesbibliothek als Teil des festen Wandschmucks im Neubau aus Baumitteln angekauft worden. Heute wird das Werk im Magazin aufbewahrt.

Die Aktenlage zu diesen Werken ist lückenhaft. Ersichtlich ist, dass das Finanzministerium dem Ankauf des Wandteppichs und der beiden Pastelle erst nachträglich zugestimmt hat. Offenbar ergaben sich auch Fragen bezüglich des Verfahrens beim Ankauf der anderen "Ausstattungsgegenstände“, denn der Rechungshof bat in seiner Feststellung vom 31.7.1969 um eine Begründung "ob vor oder bei der Beschaffung der Kunstwerke die von der Oberfinanzdirektion zu bestellende Kunstkommission beratend tätig war und ob die zuständigen Fachverwaltungen entsprechend der Bekanntmachung der Landesregierung Baden-Württemberg vom 2.5.1955 bei den getätigten Ankäufen mitgewirkt haben." (Staatsarchiv Ludwigsburg EL403, Nr. 2821, NordWürtt.).

In der Stellungnahme der Oberfinanzdirektion Stuttgart vom 16. Februar 1970 (Staatsarchiv Ludwigsburg, EL 403, Nr. 2821, NordWürtt.) heißt es, dass der Wandteppich und die beiden Pastellbilder in der Kartei der Kunstkommission enthalten seien, die Kunstkommission in ihrer 31. Sitzung am 30.7.1965 dem Kauf des Bildes "Monsun“ - nachträglich - zugestimmt habe, die Metalldrucke „San Marco, Venedig“ noch nicht registriert seien und der Ankauf des Buches von Henry Moore ein besonderes Anliegen der Landesbibliothek gewesen sei, da hier Kunst und Buch in besonderer Verbindung zum Ausdruck komme.

Des weiteren heißt es in dem Schreiben, „bei den erworbenen künstlerischen Arbeiten war die Zusammenarbeit zwischen Künstler und Architekt nicht in Zusammenhang mit der Werkplanung erforderlich. Es handelt sich bei den Ankäufen um bewegliche Werke, die im Neubau erst nach dessen Fertigstellung gehängt werden können.....

Die Werke von Abakanowicz, Camaro und Nesch wurden im Bereich der Direktion, abseits vom zentralen Treppenhaus, gehängt. Die Platzierungen entsprechen nicht den qualitätvollen Arbeiten und vermitteln eher den Eindruck von Zufälligkeit. Die Kunstwerke benötigten weitere und größer dimensionierte Wände und verlieren durch deren - an sich schönen - Backsteine an Strahlkraft. 


\section{Kunstwerke im Außen- und Innenraum}

Ende 1968 trafen sich Architekt Linde und Bibliotheksdirektor Hoffmann zu einem Gespräch über die künstlerische Ausstattung der Landesbibliothek. Linde war besonders an der Gestaltung der Grünanlagen vor der Bibliothek gelegen. So wandte er sich am 13.12.1968 an Bibliotheksdirektor Hoffmann: „...müßte ich Sie bitten, sich die Zeit zu nehmen, um auch die Umgebung der Bibliothek mit uns zu besprechen, denn die Außenräume der Bibliothek sind als Vorbereiche gleich ernst zu nehmen wie die Innenräume selbst. Es liegt mir sehr daran, einen Anziehungspunkt zu schaffen, der die Menschen in die Nähe der Bibliothek lockt, damit diese in das öffentliche Leben gut eingebunden ist." (HStA, Q 3/39a, Bü 84).

Professor Linde schlug für das Vorgelände der Bibliothek eine Plastik des Berliner Bildhauers Bernhard Heiliger vor, für die Innenausstattung brachte die Bibliothek Werke von HAP Grieshaber und Josua Reichert ins Gespräch.

Diese Vorschläge wurden offenbar in der Kunstkommission besprochen und genehmigt, wie es dem Schreiben des Finanzministeriums an das Staatliche Hochbauamt I vom 23. Januar 1970 (IV A 350 d -186/70) zu entnehmen ist:

„Die Kunstkommission bei der Oberfinanzdirektion Stuttgart hat in ihrer 41. Sitzung am 14. Oktober dem Vorschlag zugestimmt,

a) die Plastik Montana von Professor Bernhard Heiliger zum Preis von 95.000 DM zuzüglich Mehrwertsteuer und Transportkosten anzukaufen und

b) den Grafiker Josua Reichert im Rahmen der noch verfügbaren Mittel mit Farbgrafiken im Innenraumbereich zu beauftragen.

Die Kunstkommission geht davon aus, daß die Direktion der Landesbibliothek zugestimmt hat.

Im Auftrag

gez. Fecker"

\section{Bernhard Heiliger, „Montana I“ 1968, (Bronze teilweise poliert, $165 \times 305 \times 100 \mathrm{~cm}$ )}

Bernhard Heiliger (geb. 11. November 1915 in Stettin, gest. 25. Oktober 1995 in Berlin) gehörte zu den bedeutendsten Künstlern der Nachkriegszeit, den die FAZ am 10. Juli 1995 als "auch im Ausland bekannten Bildhauer der Bundesrepublik, ein Staatskünstler fast, wie die öffentlichen Aufträge ... bezeugen" würdigte. Baden-Württemberg hatte bereits 1961 eine Bronzeplastik Heiligers für das Neue Schloss in Stuttgart erworben. Nun sollte vor der neuen Landesbibliothek, in Sichtweite zum gegenüberliegenden Schloss, eine weitere Arbeit des Bildhauers aufgestellt werden. Die Wahl fiel auf "Montana I", einer weit auseinander schwingenden Bronzeplastik, die die Assoziation eines Buches aufkommen lässt und auf den dahinter aufragenden Lesesaal weist.

Für die künstlerische Ausgestaltung des öffentlich zugänglichen Innenraums wurde Josua Reichert, ein Schüler von HAP Grieshaber, beauftragt. Dem Schriftwechsel zwischen der Landesbibliothek und Grieshaber ist zu entnehmen, dass zunächst Werke Grieshabers im Gespräch waren. Grieshaber, der nach einer Schriftsetzerlehre u.a. in Stuttgart bei Professor Schneidler Kalligraphie studiert hatte, war während des Zweiten Weltkriegs mit Berufsverbot belegt worden. Nach dem Krieg entwickelte er den Holzschnitt zum eigenständigen, monumentalen Wandbild, indem er eine überzeugende Synthese des - mittelalterlichen Linienschnittes mit dem Flächenholzschnitt schuf. Nach documenta-Teilnahmen erhielt er zahlreiche Aufträge für öffentliche Bauten, bei denen er den Holzschnitt zu Großbildern monumentalisierte. Besonders mit seinen großen Bildkompositionen und Themen, die die allgemeinen Bedrängnisse und Nöte der Zeit aufgriffen, erlangte Grieshaber eine Popularität wie kein anderer deutscher Künstler seiner Zeit.

Die Landesbibliothek hatte sich schon seit längeren intensiv um Grieshabers künstlerische Mitwirkung bemüht. Am 21. August 1968 schrieb Professor Hoffmann an Professor Linde: „[...] Meine Mitarbeiter und ich sind der Auffassung, daß diese sieben großen griechischen 
Buchstaben [der Prometheus-Folge] etwas besonders geeignetes für die neue Landesbibliothek sind... Die Entscheidung können sicher nur Sie treffen."

Bei der Prometheus-Folge handelte es sich um farbige Stoffdrucke, die bei der Firma pausa in Mössingen bei Tübingen in einer Auflage von je acht Stück hergestellt wurden. Aus der lückenhaft überlieferten Korrespondenz wird ersichtlich, dass die Oberfinanzdirektion und wohl auch die Kunstkommission den Erwerb der Stoffbahnen ablehnten, weil keine Einigkeit bezüglich der Haltbarkeit und Hängung der Stoffdrucke bestand.

Die Kunstkommission stimmte zu, Josua Reichert mit der Ausgestaltung mit farbigen Grafiken zu beauftragen und dafür Mittel von 50.000 DM zur Verfügung zu stellen.

\section{Josua Reichert, Die Stuttgarter Drucke, 1971 - 1973}

Reiberdruck. Holz-, Blei- und Kunststofftypen, Lynolschnitte, PVC-Schnitte.

Josua Reichert (geb. Juni 1937 in Stuttgart, lebt in Haidholzen) jobbte zunächst in einer Druckerei und studierte 1959 an der Kunstakademie in Karlsruhe bei HAP Grieshaber, der inn "ohne vorankündigung ins säure- oder wechselbad der typografie warf." Von da an arbeitete Reichert, der sich halb als Maler, halb als Drucker, keineswegs als Typograph verstand, in eigenen Ateliers in Stuttgart und München. 1960 war er als Vertreter „eines neuen Expressionismus" in der Ausstellung des literarischen Expressionismus im SchillerNationalmuseum Marbach am Neckar mit Textbildfahnen - überlebensgroße Blätter mit Gedichten von Benn, Heym, Trakl, Stadler, Lasker-Schüler - vertreten.

Reicherts Arbeiten und Ideen fielen Bibliotheksdirektor Hoffmann auf, und die WLB erwarb die ersten Blätter von Reicherts Mappenwerken, 1965 dann auch den "codex typographicus“. „[...] die herren von der landesbibliothek hatten mich von anfang an, also seit 1960 wie einen splitter im auge. sie kauften die ersten blätter... [...] das „projekt“ war die ausschmückung des geplanten neubaus der bibliothek. es war der erste bau, der mir angeboten, für meine drucke zur verfügung gestellt wurde, es gab inn aber noch nicht."

An das „Projekt“ der Stuttgarter Drucke erinnert sich Josua Reichert: Als Student habe er die „Kunst am Bau“ verachtet, weil er sie für zu konventionell und einengend sah. Deshalb habe inn die Bedingung, dass ein Kunstwerk fest mit dem Bau verbunden sein muss, bei der ersten Besprechung für die Landesbibliothek „fast in die Knie gezwungen“. Er sah Glasfenster, Graffiti, Mosaike, Fußböden, Fresken, angestrichene Betonwände, aber keine Drucke an den Wänden. Schließlich sei es ihm gelungen, die Kunstkommission zu überzeugen, dass ein großer Druck, verglast und eingerahmt, allein durch sein Gewicht fest mit dem Bau verbunden sein wird.

Reichert erinnert sich an die Klagen der „bibliothekare“, daß die Architektur des Neubaus schwierig sei und dass es keine Wände gäbe, was inn - Reichert - aber nicht weiter störte. Der Lesesaal etwa, dessen Betondecke von Stützen getragen wird, war für Reichert kein Problem. Inspiriert von Besuchen der Hagia Sophia und osmanischer Moscheen in der Türkei hatte er schon die Lösung vor Augen.

Was die Wahl der Texte betraf, hatte Reichert völlig freie Hand. Er erarbeitete ein Konzept, indem er auf schon vorhandene Arbeiten mit Texten zurückgriff, und stellte es der „kommission“" vor:

„else lasker-schüler: fünf gedichte aus den „hebräischen balladen“, jakob, esther, boas, ruth, saul; von georg trakl: drei herbstgedichte; sechs buchstaben: hebräisch, arabisch, griechisch, kyrillisch, lateinisch und ein egyptienne-X; vier große typografische figuren aus dem garten des polyphem; drei sprüche:sator, media vita, a rose is a rose....; zwei psalmen: 23 und 120; zwei typografische kompositionen; prosa: lessing, kafka; lyrik: hölderlin, greve." Der Vorschlag 
wurde angenommen.

Josua Reichert begann sein „work in progress“: „die liste veränderte sich. ich war inmitten eines strudels, um mich kreisten die buchstaben, die dichter, die schriften, die bücher und fotos. in england bestellte ich holztypen, in kirchheim ließ ich ebenfalls schriften schneiden, ein intarsienschneider sägte in münchen nach meinen zeichnungen typen aus plexiglas aus, ich kaufte metallettern, schnitt aus pvc aus, zersägte holzleisten.... ich lernte die alphabete, studierte die schriften, die buchstaben, lernte nachts die regeln der arabischen und hebräischen typografie, studierte die kanons, las, zeichnete, erfand und verwarf, sah manchmal im geiste schon die werke, von denen ich noch nicht einmal wußte, wie ich sie drucken kann...."

Eine aus 20 Personen bestehende Kommission entschied - noch ohne Vorliegen der Arbeiten - über deren Platzierung. Die Lieferung erfolgte dann in zwei Teilen: am 15. Juni 1971 und am 3. Mai 1973. Nachdem die Blätter einheitlich mit Aluprofilen gerahmt wurden, bestimmten Künstler, Architekt und Hausherr - Josua Reichert, Professor Horst Linde und der neue Bibliotheksdirektor Dr. Hans-Peter Geh - die letztendlich gültige Hängung. Die sechs Buchstaben, denen die Besucher auf der Rampe vom Eingang Konrad-Adenauer-Straße hinauf zu Garderobe und Treppenhaus begegnen, bilden den Ausgangspunkt für Reicherts Konzept: "jeder buchstabe sollte ein keimling sein, aus dem verschiedenartigste typografie hervorkommt. gedichtblätter, prosaseiten, sprüche, wörter, sätze, palindrome, typenkonstellationen, buchstabenbilder, kompositionen. von hier aus sollten diese sich durch das gebäude verbreiten, ich dachte gleich weiter, dachte an die umliegenden bauten, an die gebäude in der stadt, an gebäude in anderen städten......

Ich habe in den folgenden 25 jahren an dieser ausbreitung gearbeitet und weitere 200 große drucke in bibliotheken und anderen bauwerken [Anm: u.a. Universitätsbibliotheken Augsburg, Erlangen-Nürnberg, Regensburg und Würzburg] untergebracht. der aufmerksame betrachter findet überall die alten bekannten und freunde aus der landesbibliothek wieder!“

Die übrigen Blätter wurden im Treppenhaus, auf der Informationsebene und im Hauptlesesaal aufgehängt. Am oberen Eingang befinden sich das „Hebräische Alphabet“ und das „Zionslied von Jehuda Halevi“. Auf der Treppe begrüßt die Besucher das Wortgedicht „SATOR AREPO TENET OPERA ROTAS“, gegenüberliegend das Gedicht „A rose is a rose is a rose is a rose“ von Gertrude Stein. Steigt der Besucher die Treppe hinauf, stößt er auf Friedrich Hölderlins Gedicht „An die Parzen“, das in großer Höhe an einem der Betonpfeiler befestigt ist. Am Geländer des Treppenhaus stehen sich weitere Gedichte gegenüber: Hölderlins Fragment „Wenn über dem Weinberg es flammt..." und „Melancholie“ sowie „Im Park“ von Georg Trakl.

Im Hauptlesesaal findet sich das Schrift-Bild „Boas“, ein Gedicht von Else Lasker-Schüler. Besonders markant aber ist das runde Bild „Komposition mit $Y$ und a“, das an einem der Pfeiler im Lesesaal hängt. Dieses Motiv hat Josua Reichert auf seiner Reise in der Türkei aufgegriffen und erinnert an die runden Schilde in der Hagia Sophia oder einer osmanischen Moschee.

Im Informationsbereich, neben dem großartigen Porträt des Bibliotheksgründers Carl Eugen, Herzog von Württemberg, das $1753 / 55$ von Pompeo Batoni gemalt wurde und zum Stiftungsgut gehörte, findet sich der Druck „media vita in morte sumus“. Von Martin Luther übersetzt, lautet der Anfang des Antiphons über den Tod von Notker dem Stammler (geb. um 840 bei St. Gallen, gest. 912): "Mitten im Leben sind wir mit dem Tod umfangen..." Dieser Text war Reicherts Leitmotiv für seine „Stuttgarter Drucke“, den er gerne am Eingang von der Konrad-Adenauer-Straße gesehen hätte.

Der Maler und Grafiker Josua Reichert hat mit seinen Drucken ein unverwechselbares Oeuvre geschaffen. Für die Herausforderung „Kunst am Bau“ der neuen Landesbibliothek beschritt er 
neue Wege, indem er monumentale Schrift-Bilder entwarf und selbst druckte. Seit nahezu 40 Jahren prägen diese Schrift-Bilder die Württembergische Landesbibliothek und unterstreichen deren offene und großzügige, demokratische Architektur in den Publikumsbereichen. Josua Reicherts „Stuttgarter Drucke“ sind untrennbar mit der Württembergischen Landesbibliothek verbunden.

\section{Die Stuttgarter Drucke}

Bogen $1-6(120 \times 100 \mathrm{~cm})$

1) Hebräisches Mem

2. Arabisches Vav

3) Griechisches Psi

4) Lateinisches $P$ (mitQ)

5) Kyrillisches Jat

6) Egyptienne V

Bogen $7(120 \times 100 \mathrm{~cm})$

Hebäisches Alphabeth

Bogen $8(160 \times 100 \mathrm{~cm})$

Zionslied von Jehuda Halevi

Bogen 9 und 10 (je $200 \times 120 \mathrm{~cm}$ )

Psalm 122: Herrlichkeit Jerusalems

Bogen 11 und $12($ je $120 \times 120 \mathrm{~cm})$

Alpha und Omega

Bogen $13(170 \times 120 \mathrm{~cm})$

Fragment eines Gedichts von Alkaios

Bogen $14(120 \times 240 \mathrm{~cm})$

Fragment eines Gedichtes von Sappho

Bogen $15(160 \times 120 \mathrm{~cm})$

SATOR AREPO TENET OPERA ROTAS

Antiker Spruch

Bogen $16(210 \times 120 \mathrm{~cm})$

MEDIA IN VITA MORTE SUMUS

(In Übersetzung Martin Luthers: Mitten im Leben sind wir mit dem Tod umfangen)

Bogen 17 und $18(120 \times 190 \mathrm{~cm}, 115 \times 170 \mathrm{~cm})$

Rubaij von Muhammad Schamsuddin Hafis

Bogen $19(120 \times 170 \mathrm{~cm})$

Rubaij von Farid ad - Din Attar

Bogen $22(100 \times 120 \mathrm{~cm})$

An die Parzen

Ode von Friedrich Hölderlin

Bogen $23(120 \times 100 \mathrm{~cm})$

Hälfte des Lebens

Gedicht von Friedrich Hölderlin

Bogen $24(120 \times 250 \mathrm{~cm})$

Fragment [No 50] von Friedrich Hölderlin

Bogen $25(100 \times 120 \mathrm{~cm})$

Friedensahnung

Gedicht von Oskar Loerke 
Bogen $26(135 \times 120 \mathrm{~cm})$

a rose is a rose is a rose is arose

von Gertrude Stein

Bogen $27(120 \times 280 \mathrm{~cm})$
Kyrillischer Typenbogen

Bogen $28(120 \times 200 \mathrm{~cm})$

Melancholie

Gedicht von Georg Trakl

Bogen $29(120 \times 200 \mathrm{~cm})$

Im Park

Gedicht von Georg Trakl

Bogen 30

Boas

Gedicht von Else Lasker-Schüler

Bogen $31(200 \times 120 \mathrm{~cm})$

Die Bäume

Parabel von Franz Kafka

Bogen 32, 33, 34, 35, 36 (Durchmesser $100 \mathrm{~cm}$ )

Fünf runde Bilder

1) Komposition mit $Y$ und a

2) Komposition mit y und a

3) Komposition mit $Y$ und a

4) Komposition mit $Y$ und a

5) Komposition mit y und $\mathrm{J}$

Technische Angaben:

- Farbe: handelsübliche Buchdruckfarbe

- Papier: 250 g/qm² schwerer, ungeleimter Kupferdruckkarton, holzfrei.

- Die verwendeten Schriften sind alle in Holz oder Kunststoff geschnitten. Alle Figuren und sonstigen Elemente wie Leisten und Platten sind ebenfalls aus Holz oder Kunststoff.

- $\quad$ Alle Bogen (bis auf No. 22, 23, 25) sind mit einem Löffel durchgerieben.

- Von allen Drucken existieren 3 Abzüge; gedruckt wurden sie 1971/72 in München.

\section{Literatur:}

Kunst in der Architektur Land Baden-Württemberg. 25 Jahre künstlerisches Schaffen im staatlichen Hochbau in Baden-Württemberg. Hrsg. Finanzministerium Baden-Württemberg, Stuttgart 1979.

Kunstkonzeption des Landes Baden-Württemberg. Staatsministerium Baden-Württemberg, Stuttgart 1990.

Literaturhinweis zu Josua Reichert:

Die Stuttgarter Drucke von Josua Reichert. Hrsg. von der Württembergischen

Bibliotheksgesellschaft, Stuttgart 1972.

Josua Reichert. Werkverzeichnis 1959 - 1995. Mit Anmerkungen des Künstlers zu seinen Werkgruppen. Bearbeitet von Waltraud Pfäfflin und Klaus Maurice, Stuttgart 1997.

Der leicht veränderte Beitrag ist erschienen in: La REVUE de la BNU. Bibliothèque nationale et universitaire de Strasbourg, Printemps 2010 / Numéro 01. 